\title{
¿Nanomateriales o nanopartículas? Desafíos de la ontología del dominio nano*
}

\section{Nanomaterials or nanoparticles? Challenges of the nano domain ontology}

\author{
Alfio Zambon, ${ }^{* *}$ Mariana Córdoba ${ }^{* * *}$ y Olimpia Lombardi***, 1
}

\begin{abstract}
The aim of this article is to formulate some philosophical problems resulting from the analysis of the field of nanosciences, taking certain discussions coming from the philosophy of chemistry as the departing point. Many problems, usually approached to in their epistemological dimension in the philosophy of science, are endowed with a relevant ontological dimension that should not be disregarded. On the basis of this belief, we are particularly interested in the problems related to the ontology of nanosciences, specifically: Of what kind of entities is the nanoworld populated? Which is the nature of those entities? How can this domain be characterized and understood? With this purpose, we will analyze how nanomaterials or nanoparticles can be characterized and what place these entities occupy in the chemical world. In particular, we will argue that, in the level of nano-chemistry, the ontological categories of individual and stuff are not adequate, and a new category is required, whose intermediate nature is manifested in language: we use the term 'nanoparticles' when we think in individuals, whereas we talk of 'nanomaterials' when we think in stuff.
\end{abstract}

KEYWORDS: nanoparticles, nanomaterials, individuals, stuff, ontology.

RESUMEN: En este artículo nos proponemos dejar planteados algunos problemas filosóficos que surgen del análisis del campo de las nanociencias, partiendo de ciertas discusiones provenientes de la filosofía de la química. Muchos problemas, que suelen abordarse solo en su dimensión epistemológica en la filosofía de las ciencias, presentan una importante dimensión ontológica que no debe desatenderse. Sobre la base de esta convicción, nos interesa especialmente abordar problemas relativos a la ontología de las nanociencias, en particular: ¿de qué tipo de entidades está poblado el nanomundo?, ¿cuál es la naturaleza de dichas entidades?, ¿cómo puede caracterizarse y comprenderse este dominio? Con este propósito, analizaremos de qué modo pueden caracterizarse los nanomateriales o nanopartículas y cuál es el lugar que estas entidades ocupan en el mundo químico. En particular, se argumentará que al nivel de la nanoquímica las categorías ontológicas de individuo y de materia no son adecuadas, y se requiere una nueva categoría, cuya naturaleza intermedia se manifiesta en el lenguaje: usamos el término 'nanopartículas' cuando pensamos en individuos, mientras que hablamos de 'nanomateriales' cuando pensamos en materia.

Recibido: 13 de octubre de 2018

Aceptado: 5 de diciembre de 2018 .

* Deseamos agradecer la invitación a participar en el encuentro Segunda Jornada Nacional y Primera Regional de Bio-Nanotecnología, realizado en la Universidad Nacional de Quilmes, los días 4 y 5 de octubre de 2018. El presente trabajo se basa en la ponencia presentada en dicho encuentro.

** Universidad Nacional de la Patagonia San Juan Bosco.

*** CONICET, Universidad de Buenos Aires; Argentina.

Correos electrónicos: alfiozambon@gmail.com; mariana.cordoba.revah@gmail.com

${ }^{1}$ Autora de correspondencia: olimpiafilo@gmail.com 
PALABRAS CLAVE: nanopartículas, nanomateriales, individuos, materia, ontología.

\section{Introducción}

La filosofía de la química es una disciplina joven, que ha sabido reformular y reinterpretar muchos problemas filosóficos tradicionales y muchos debates clásicos. Especialmente, esta disciplina se ha preguntado cuál es el estatuto epistemológico de la química en relación con la física, rediscutiendo argumentos clásicos y proponiendo nuevas miradas respecto de la posibilidad de la reducción de la química a la física, la emergencia y la superveniencia.

En este trabajo nos proponemos dejar planteados algunos problemas filosóficos surgidos del análisis del campo de las nanociencias, partiendo de ciertas discusiones provenientes de la filosofía de la química. Muchos problemas que suelen abordarse solo en su dimensión epistemológica en la filosofía de las ciencias presentan una importante dimensión ontológica que no debe desatenderse. Sobre la base de esta convicción, nos interesa especialmente abordar problemas relativos a la ontología de las nanociencias, en particular: ¿de qué tipo de entidades está poblado el "nanomundo", ¿cuál es la naturaleza de dichas entidades?, ¿cómo puede caracterizarse y comprenderse este dominio? Con este propósito, analizaremos de qué modo pueden caracterizarse los nanomateriales o nanopartículas y cuál es el lugar que estas entidades ocupan en el mundo químico.

\section{¿Qué se entiende por categoría ontológica?}

En la filosofía de la química se ha debatido recientemente acerca del estatus ontológico de los ítems propios de la disciplina química (Ruthenberg y van Brakel, 2008; Lewowicz y Lombardi, 2013). En este marco, se ha discutido acerca de qué categoría ontológica puede resultar más apropiada para comprender las entidades químicas. Antes de ingresar en este debate, es necesario aclarar qué se entiende, en este contexto, por 'categoría ontológica'.

Adoptaremos la tradicional idea filosófica de que las categorías ontológicas son responsables de estructurar la realidad en tanto la configuran y constituyen imponiéndole determinada forma. También de las categorías ontológicas depende cómo usamos el lenguaje, cómo percibimos y conocemos los ítems que habitan el mundo (Lewowicz y Lombardi, 2013). Las categorías ontológicas no son propiedades, no deben confundirse con taxa, ni son conceptos de clase. Las categorías ontológicas son anteriores a las taxa y a los conceptos de clase, tanto en un sentido lógico como en un sentido ontológico. El rol de las taxa y conceptos de clases es clasificar individuos, de manera que su utilización presupone la adopción de una ontología habitada por individuos. Pero las categorías ontológicas son las responsables de determinar qué tipos de cosas habitan el mundo, es decir, por qué tipo de ítems está constituida la realidad, por ejemplo, si la realidad consta de individuos 
con propiedades, si solo hay propiedades, eventos, procesos, por citar algunos.

Las categorías ontológicas determinan cuál es la estructura de cada dominio científico, en tanto imponen una determinada forma a lo real. De este modo, los ítems de un dominio dado pueden ser capturados por las categorías formales de un lenguaje, en tanto el lenguaje está, asimismo, organizado, estructurado por aquellas mismas categorías. Por ejemplo, una ontología estructurada con las categorías aristotélicas de sustancia y atributos (esenciales y accidentales) es recogida por enunciados en los que es posible distinguir el sujeto de predicación de las predicaciones. Si prestamos atención al rol determinante de las categorías ontológicas, podemos comprender la posibilidad de que exista un discurso significativo sobre un determinado dominio científico y, en consecuencia, la posibilidad de realizar inferencias exitosas respecto de lo que ocurre en él.

\section{Individuos versus stuff}

Joachim Schummer establece una interesante distinción entre lo que denomina la perspectiva de la forma y lo que llama la perspectiva de la materia (Schummer, 2008). De acuerdo con la perspectiva de la forma, el mundo es comprensible según una metafísica matematizable; se apela a las propiedades geométricas espaciales para describir los cuerpos. Por el contrario, la perspectiva de la materia se interesa por la composición de los cuerpos, por los materiales particulares de los cuales los cuerpos están compuestos. Ahora bien, el problema de la ontología de la química puede ser pensado no solo a partir de la distinción entre perspectivas epistémicas contrapuestas, sino entre categorías ontológicas diferentes (Lewowicz y Lombardi, 2013). De acuerdo con esta última mirada, la ontología de la física es una ontología de individuos y propiedades, mientras la ontología de la química es una ontología de stuff. ${ }^{2}$ Para comprender esta distinción entre dos ontologías, es necesario tener en cuenta que aquí asumimos que dos ontologías son diferentes en tanto son resultado de - en tanto están constituidas por- diferentes categorías ontológicas.

Nos referimos, entonces, por un lado, a una ontología de individuos con propiedades y, por otro, a una ontología de stuff, en tanto individuo y materia (stuff) son dos categorías ontológicas distintas.

\section{Individuos}

¿Cómo ha caracterizado la filosofía contemporánea la categoría ontológica de individuo? Para que existan individuos debe existir un principio de individuación que permita distinguir cualquier individuo de cualquier otro indi-

${ }^{2}$ El término stuff en el presente trabajo será utilizado en inglés o en ocasiones será traducido por 'materia'. Qué entendemos por stuff será elucidado en lo que sigue. 
viduo (French y Krause, 2006). En general, el principio de individuación se identifica con (o implica) la posición espacio-temporal. Las propiedades espacio-temporales juegan un papel fundamental en la individualidad, porque dos individuos no pueden ocupar al mismo tiempo la misma ubicación espacial (impenetrabilidad).

El compromiso con la existencia de individuos implica la aceptación de una ontología de individuos y propiedades. En la filosofía occidental, los individuos se conciben como el sustrato en el que las propiedades inhieren y como aquello que confiere unidad a la multiplicidad de las propiedades. A su vez, las propiedades son o bien esenciales (las propiedades responsables de que el individuo pueda ser re-identificado a través del tiempo a pesar de los cambios de propiedades que sufre), y accidentales (aquellas que pueden cambiar en el transcurso del tiempo).

Se afirma que un individuo es una entidad 'completa' porque es indivisible. Esto significa que o bien no puede ser dividida o bien, si puede serlo, de la división resultan individuos diferentes del individuo original. A su vez, el individuo obedece a la categoría kantiana de cantidad (unidad-pluralidad), de modo que los individuos o bien son uno (cada individuo), o bien son muchos, es decir, una pluralidad de individuos. Dado un grupo de individuos, estos pueden ser contados, porque tienen su propia individualidad y se los puede volver a identificar dentro del grupo. Cuando los individuos son agrupados de acuerdo con sus propiedades - cuando son clasificados- las agrupaciones resultantes son clases, algunas de las cuales se consideran naturales.

Dado que las categorías son responsables de la estructura tanto de la realidad como del lenguaje, la categoría de individuo tiene un correlato lingüístico en los términos singulares y en los sujetos lógicos de las proposiciones (Strawson, 1959; Tugendhat, 1982). La ontología de individuos y propiedades es la que subyace a la mayoría de los lenguajes cotidianos, y también a la mayor parte de los sistemas lógicos. La perspectiva de un mundo de individuos y propiedades es la visión dominante en el pensamiento filosófico tradicional de occidente.

Stuff

La ontología de la macro-química, en cambio, se entiende mejor como una ontología de stuff (materia). Como señalan Klaus Ruthenberg y Jaap van Brakel (2008), las sustancias químicas son stuff. Pero la categoría de stuff no resulta tan clara pues, como sostiene Schummer, no existe una fuerte tradición científica para ayudarnos a comprenderla. Para caracterizarla, resulta claro compararla con la categoría de individuo. Al igual que en el caso de los individuos, debe existir un principio que distinga un stuff de otros, pero dicho principio nada tiene que ver con el espacio y el tiempo. Sin embargo, porciones de stuff existen en el espacio y en el tiempo (una porción de agua puede ubicarse espacio-temporalmente, por ejemplo, ahora, en mi vaso). A diferencia de los individuos, una porción de stuff puede dividirse en porciones del mismo stuff, 
pero una materia en particular no es una mera adición de sus porciones. Un stuff es uno y múltiple; por ejemplo, una única sustancia química constituye una unidad, pero a la vez es múltiple en la medida en que posee múltiples manifestaciones bajo la forma de porciones del mismo stuff.

Sin embargo, los agregados de porciones de stuff no se comportan como lo hacen las agrupaciones o clases de individuos. Por un lado, dado un agregado de porciones de stuff, tales porciones no pueden ser reidentificadas en el agregado: una vez que el agregado se ha formado, no puede decirse que esta es una de las porciones y aquella, la otra; por ejemplo, una vez que se han mezclado dos porciones de agua en un recipiente, no se las puede reidentificar en la mezcla. Por otro lado, y como consecuencia de lo anterior, dado un agregado de porciones de stuff, estas no pueden ser contadas en el agregado: el agregado de dos porciones de un cierto stuff $X$ no equivale a "dos X", sino a "más X"; si agrego una porción de agua a otra porción de agua, no tengo "dos aguas" ni puedo contar las dos porciones de agua en el agregado.

Esta perspectiva, que parte de la distinción entre individuos y stuff, desde un punto de vista ontológico, es muy útil para abordar ciertos problemas filosóficos que surgen de la consideración de las disciplinas científicas. Insistimos en que debe tenerse en cuenta que esta perspectiva no refiere a dos enfoques epistémicos diferentes, sino a dos categorías ontológicas distintas. Individuos y stuff son dos categorías ontológicas básicas, responsables de la estructura fundamental del mundo físico y del mundo macroquímico, respectivamente.

\section{La posición intermedia del dominio nano}

Lucía Lewowicz y Olimpia Lombardi (2013) sostienen que, así como la física se ocupa de individuos con propiedades, la química se ocupa de stuff. Ahora bien, el análisis de la ontología de la química como una ontología de materia o stuff es sumamente apropiado; sin embargo, en esta caracterización no hay lugar para los nanomateriales. La categoría ontológica de stuff es adecuada para comprender las sustancias químicas macroscópicas tradicionales, pero no resulta igualmente satisfactoria si pretendemos utilizarla para comprender el dominio nano. ¿Por qué? Los nanomateriales manifiestan propiedades químicas y físicas peculiares. Esas propiedades son muy interesantes no solo desde un punto de vista teórico, sino también en la aplicación a campos tan diferentes como el diseño de nuevos materiales, la electrónica, la síntesis de nuevos catalizadores o la creación de dispositivos de liberación de fármacos.

¿Qué categoría ontológica resulta adecuada para pensar la naturaleza de los nanomateriales? ¿Qué tipo de entidades son los nanomateriales? Cuando tenemos en cuenta los nanomateriales, las categorías de individuos y stuff se muestran problemáticas. Estas resultan insuficientes para concebir la naturaleza del nanomundo, pues los ítems que pueblan el nanomundo tienen 
ciertas características propias de los individuos, pero también algunas propias de la materia.

Como los individuos, las nanopartículas pueden ser contadas; si se dividen, se obtienen nanopartículas de un tipo diferente al original, con diferentes propiedades ópticas, magnéticas y químicas, entre otras. Los ítems en el nivel nano pueden formar un agregado en el cual pueden ser re-identificados. Pero, al igual que ocurre con los materiales (o stuff), los ítems nano tienen una propiedad química específica, la reactividad. Los nanomateriales participan en reacciones químicas, como sustancias químicas, y en esas reacciones pierden su individualidad. A pesar de esto, no son stuff en el sentido tradicional de la macro-química, dado que las propiedades de los nanomateriales cambian con el tamaño de las nanopartículas, porque dependen de las características de su superficie externa. Además, las propiedades de los nanomateriales pueden ser muy diferentes de las de la sustancia macroscópica correspondiente (Córdoba y Zambon, 2017).

Por estos motivos, se ha postulado una tercera categoría para pensar en el mundo nanoquímico: la categoría de nano-individuo (Córdoba y Zambon, 2017). Esta categoría ontológica no surge de una síntesis conceptual entre el concepto de individuo físico y el concepto de materia química; no es un artificio matemático o químico creado exclusivamente para tratar algunos problemas. Se trata de un nuevo tipo de "individuos", diferentes de las partículas individuales de la física, pero que tampoco pueden asimilarse a las sustancias químicas. De esta manera, podemos entender las peculiaridades del comportamiento químico de los nanomateriales, y concebir la nano-química como una genuina rama de la química, no como la combinación metodológica de la química macroscópica y molecular.

\section{Los dominios de la química}

Si prestamos atención a los nanomateriales, encontramos que no es posible concebir el dominio de la química como un único dominio (Córdoba, Labarca y Zambon, 2013; Zambon y Córdoba, 2017). De hecho, una sustancia química puede ser identificada por medio de sus propiedades macroscópicas o por medio de sus propiedades microscópicas. En este sentido, la macro-química y la micro-química son dos dominios diferentes. Pero si consideramos los nanomateriales, debemos añadir a esta imagen un tercer nivel, el de la nanoquímica, que constituye un estrato diferente en el dominio micro-químico (Zambon y Córdoba, 2017).

Caracterizaremos brevemente estos dominios y niveles. En el macro-dominio encontramos las sustancias que el químico manipula en el laboratorio y donde aparecen todas las propiedades físicas y químicas que se determinan experimentalmente (densidad, índice de difracción, características organolépticas, entre otras). Es el dominio de los materiales o stuff. En el micro-dominio, encontramos el nivel molecular, poblado por las estructuras químicas 
utilizadas para describir los cambios que tienen lugar en las reacciones, y para explicar muchas características y propiedades de las sustancias químicas. En este nivel, las entidades poseen una entropía mínima: las estructuras más estables son las de mínima energía.

Tradicionalmente, este micro-dominio corresponde al estudio de los grupos funcionales, los isómeros conformacionales, las características de los enlaces, los efectos de resonancia e inductivos, etc., y está habitado por individuos en el sentido físico clásico: las moléculas, con sus propiedades estructurales definidas. Ahora bien, al nivel molecular en el micro-dominio es necesario agregar el nano-nivel, constituido por ítems con características únicas, muy diferentes de las propiedades físicas y químicas que la sustancia correspondiente presentaba en los otros niveles. Los nanomateriales resultan de la reducción de partículas de una sustancia química a la escala nanométrica: mientras que las sustancias en el dominio macro son continuas y homogéneas, en la nano-escala, los átomos y sus relaciones estructurales adquieren una importancia central.

Como señalamos, desde una perspectiva ontológica los nano-ítems no pueden ser caracterizados ni como stuff ni como individuos, sino como nanoindividuos. Por lo tanto, en lugar de una imagen de química donde conviven solo dos niveles, surge una imagen con dos dominios (véase la figura 1).

FIGURA 1. El macro-dominio y el micro-dominio, pero con tres niveles, pues en el micro-dominio conviven el nivel molecular y el nivel nano, constituidos por categorías ontológicas diferentes.

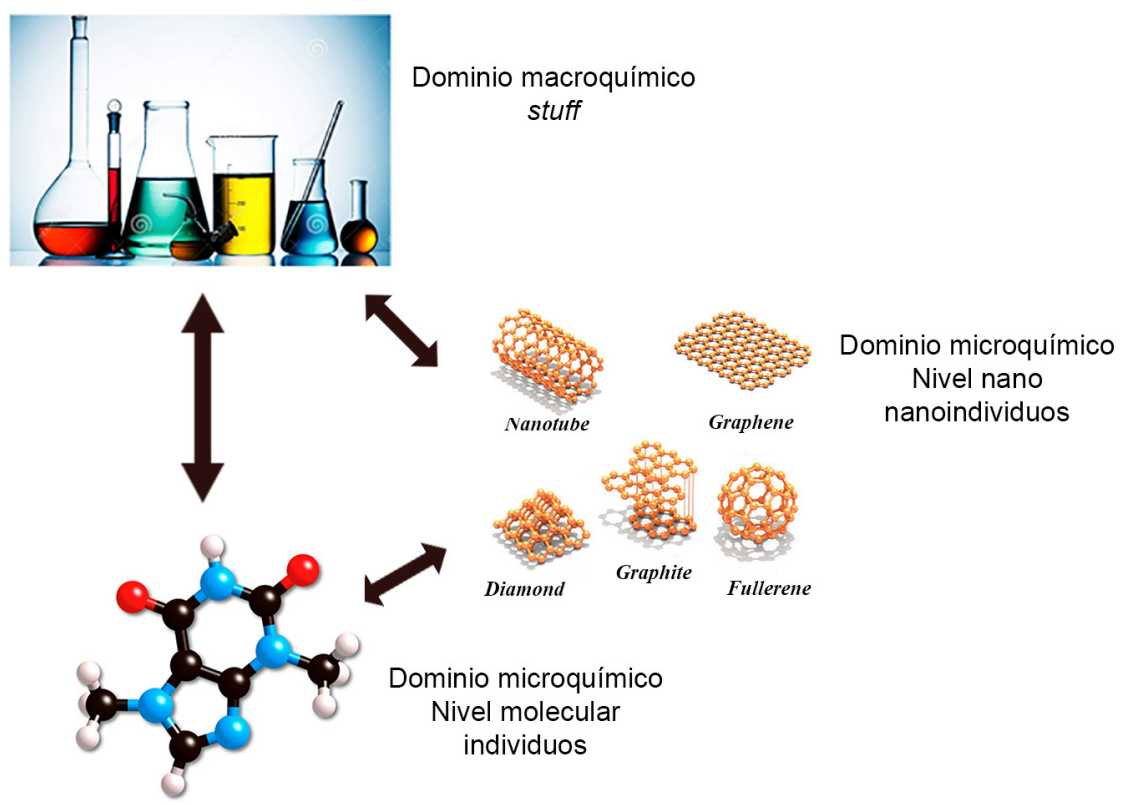

Fuente: Elaboración de los autores. 
En síntesis, las entidades del dominio de la macro-química se caracterizan ontológicamente como stuff; el dominio micro-químico se subdivide en el nivel molecular, poblado por moléculas individuales (individuos), y el nano nivel, habitado por nano-individuos, que son ítems con sus propias peculiaridades. De esta manera, si consideramos que la macro-química, la química molecular y la nano-química constituyen tres niveles diferentes, el nivel de la nano-química se puede caracterizar como un nivel de naturaleza "intermedia" dadas las características ontológicas de los ítems que lo habitan. Cabe destacar que esta suerte de naturaleza intermedia de los nanomateriales -entre individuos y stuff-, se manifiesta de manera clara en el lenguaje: usamos el término "nanopartículas" cuando pensamos en individuos, mientras que hablamos de "nanomateriales" cuando pensamos en stuff.

\section{Consideraciones finales}

El reduccionismo científico, que propugna la reducción de la química a la física - en particular, a la mecánica cuántica - supone que las leyes y conceptos del campo a ser reducido pueden ser completamente reemplazados por (deducidos y definidos a partir de) las leyes y conceptos del campo reductor. Pero debe advertirse que este supuesto de reducción suele adoptar implícitamente la convicción de que o bien los dominios reducido y reductor constituyen la misma ontología, o bien el dominio reducido no está poblado por entidades reales, sino por ítems aparentes o meramente fenoménicos. Este supuesto de carácter ontológico suele operar como fundamento y justificación de aquél, de carácter epistémico, acerca de la posibilidad de deducción de las leyes del dominio reducido a partir de las del dominio reductor.

$\mathrm{Si}$ aceptamos que la física se ocupa de individuos, mientras que la química se ocupa de stuff, ya no resulta tan sencillo asumir dichos compromisos reduccionistas. Si además consideramos que el dominio de la química es, a su vez, más complejo, dado que el nivel de la química molecular da lugar nuevamente a los individuos y el nano-nivel a entidades que hemos caracterizado como nano-individuos, la situación es todavía más desafiante.

La nanociencia generalmente se define en términos de una escala de longitud: se considera que es la ciencia que estudia las partículas entre 1 y 1000 nanómetros (Cao, 2004; Gago, 2010), que es el estudio de aquellas cosas que se encuentran a medio camino entre las escalas molecular y microscópica. Aquí nos hemos propuesto pensar la nanociencia de una manera diferente, no simplemente en términos del tamaño de los ítems estudiados, sino teniendo en cuenta la naturaleza ontológica de esos objetos.

Nuevos problemas surgen si comenzamos a analizar las nanociencias desde una perspectiva filosófica, y viejos problemas adquieren nuevas dimensiones y exigen una reformulación ¿Es posible hablar de reducción dentro de la química, entre dominios estructurados de acuerdo con diferentes categorías ontológicas? ¿Cuál es la relación que puede establecerse 
entre la macro-química y la micro-química, si consideramos que esta última contiene dos niveles diferenciados?

Una perspectiva ontológica sobre aquello a lo que refieren las disciplinas científicas, especialmente la nanociencia, puede dar lugar a una nueva dimensión de los debates filosóficos tradicionales y abrir un nuevo panorama para analizar problemas estrictamente científicos, en general anclados en supuestos tradicionales. Nuestro trabajo se propone plantear las nuevas preguntas que se imponen para una filosofía de las nanociencias.

\section{Referencias}

Cao, G. (2004). Nanostructure and Nanomaterials: Synthesis, Properties and Applications. Londres: Imperial College Press.

Córdoba, M., Labarca., M. y Zambon, A. (2013). Acerca de la unicidad de la sustancia en química. Revista Colombiana de Filosofía de la Ciencia, 26: 167-180.

Córdoba M. y Zambon, A. (2017). How to handle nanomaterials? The re-entry of individuals into the philosophy of chemistry. Foundations of Chemistry, 19: 185-196. http://dx.doi.org/10.1007/s10698-017-9283-6

French, S. y Krause, D. (2006). Identity in Physics: A Historical, Philosophical and Formal Analysis. Oxford: Oxford University Press, Oxford.

Gago, J. (2010). Nanociencia y nanotecnología. Entre la ciencia ficción del presente y la tecnología del futuro. Madrid: Fundación Española para la Ciencia y la Tecnología.

Lewowicz, L. y Lombardi, O. (2013). Stuff versus individuals. Foundations of Chemistry, 15: 65-77. http://dx.doi.org/10.1007/s10698-012-9152-2

Ruthenberg, K. y van Brakel, J. (eds.) (2008). Stuff. The Nature of Chemical Substances. Würzburg: Königshauen \& Neumann.

Schummer, J. (2008). Matter versus form, and beyond. En K. Ruthenberg y J. van Brakel (eds.), Stuff. The Nature of Chemical Substances. Würzburg: Königshauen \& Neumann, 3-18.

Strawson, P. (1959). Individuals. An Essay in Descriptive Metaphysics. Londres: Methuen. Tugendhat, E. (1982). Traditional and Analytical Philosophy: Lectures on the Philosophy of Language. Cambridge: Cambridge University Press.

Zambon, A. y Córdoba, M. (2017). Nanomaterials and intertheoretical relations: macro and nanochemistry as emergent levels. 2017-Annual Meeting of the International Society for the Philosophy of Chemistry (ISPC), París. 